\title{
Pharmacological Management of Insomnia
}

\author{
Sarika Madari ${ }^{1}$ - Raphael Golebiowski ${ }^{1} \cdot$ Meghna P. Mansukhani ${ }^{2} \cdot$ Bhanu Prakash Kolla ${ }^{1,2}$ (i)
}

Accepted: 14 January 2021 / Published online: 1 February 2021

(C) The American Society for Experimental NeuroTherapeutics, Inc. 2021

\begin{abstract}
Insomnia is a highly prevalent condition associated with significant morbidity, reduction in quality of life, and increase in healthcare costs, and is a risk factor for multiple physical and mental disorders. The primary treatment modality is cognitive behavioral therapy for insomnia (CBT-I) but this is associated with difficulties with access and higher cost as well as poor response in some patients. Therefore, pharmacotherapy for insomnia is common and hypnotic agents are among the most frequently prescribed medications in the United States. Older medications for insomnia are limited by their side effect burden and narrow therapeutic window. Newer hypnotics, on the other hand, have been shown to have a better safety profile and longer term efficacy. While some studies have shown that long-term hypnotic use is associated with adverse outcomes, the current evidence is equivocal. The decision to treat chronic insomnia disorder with long-term hypnotics should be individualized and balance the potential risks of continuing hypnotic medication use with the risks of untreated persistent insomnia and associated functional limitations. This clinical review discusses the currently available medication options to treat insomnia, their mechanisms of action, dosing, and side effect profiles. This review also provides guidance on long-term management of hypnotics and the use of these medications in the elderly, those with medical comorbidities, and other special populations.
\end{abstract}

Key Words Insomnia $\cdot$ hypnotics $\cdot$ pharmacotherapy $\cdot$ sedatives $\cdot$ sleep $\cdot$ medication $\cdot$ treatment

\begin{tabular}{|c|c|}
\hline \multicolumn{2}{|c|}{ Abbreviations } \\
\hline $\mathrm{BZ}_{1}$ & benzodiazepine receptor type I \\
\hline CBT-I & cognitive behavioral therapy for insomnia \\
\hline CYP3A & cytochrome P450, family 3, subfamily A \\
\hline DORA & dual orexin receptor antagonists \\
\hline GABA & gamma-aminobutyric acid \\
\hline $\mathrm{GABA}_{\mathrm{A}}$ & gamma-aminobutyric acid receptor type $\mathrm{A}$ \\
\hline MAOI & monoamine oxidase inhibitors \\
\hline nBBRA & $\begin{array}{l}\text { non-benzodiazepine benzodiazepine receptor } \\
\text { agonist }\end{array}$ \\
\hline OSA & obstructive sleep apnea \\
\hline OX1R & orexin receptor type 1 \\
\hline $\mathrm{OX} 2 \mathrm{R}$ & orexin receptor type 2 \\
\hline SUD & substance use disorders \\
\hline
\end{tabular}

Bhanu Prakash Kolla

kolla.bhanuprakash@mayo.edu

1 Department of Psychiatry and Psychology, Mayo Clinic, 200 First St. SW, Rochester, MN 55905, USA

2 Center for Sleep Medicine, Mayo Clinic, 200 First St. SW, Rochester, MN 55905, USA

\section{Introduction}

Insomnia is characterized by difficulty initiating and/or maintaining sleep associated with daytime impairment, not otherwise attributable to environmental deterrents to sleep such as inadequate opportunity to sleep $[1,2]$. Chronic insomnia is defined as symptoms occurring a minimum of 3 times a week for at least 3 months [1]. An estimated 30\% of the worldwide population has one or more symptoms of insomnia [3]. Insomnia can impact both physical and mental health [2]. Decreased sleep duration in association with chronic insomnia disorder is linked to increased risk of coronary artery disease, incident myocardial infarction, type 2 diabetes mellitus, obesity, systemic hypertension, and all-cause mortality [4]. Insomnia is also associated with a higher risk of incident psychiatric disorders [5]. Persistent sleep disturbance can increase the risk of depressive relapse and insomnia is known to be a significant risk factor for suicide [6, 7]. Finally, chronic insomnia disorder is a contributory factor to increasing healthcare costs and reduced quality of life [8].

Cognitive behavioral therapy for insomnia (CBT-I) is considered first-line treatment for insomnia and its efficacy is well-established [9]. CBT-I is the multimodal combination of relaxation training, cognitive restructuring, stimulus 
control, sleep restriction, and sleep hygiene educational interventions typically spanning the course of 5 weeks [10]. It has been shown to improve insomnia at least as effectively, if not better than medications, and with fewer associated adverse effects [10]. There is evidence suggesting that CBT-I may result in improvements that are more longlasting than pharmacologic treatment [11]. It is notable that in these studies, the return of insomnia symptoms was associated with medication discontinuation and not loss of efficacy. Despite the advantages of CBT-I, many providers turn to prescription hypnotics for the initial treatment of a patient presenting with insomnia [11]. Patient-based limitations to more widespread utilization of CBT-I include limited availability of trained providers as well as time and cost considerations [10]. CBT-I can be difficult to access, especially for individuals in rural locations. It can be a time-consuming and expensive intervention limiting how effectively patients with insomnia can fully engage in treatment. Additionally, some studies in recent years have shown that although CBT-I leads to improvement in sleep as evidenced by improvements in subjective sleep ratings, objective data on the positive impact of CBT-I on sleep are less robust [11]. Thus, hypnotic medications are felt to be necessary in many cases and continue to be widely prescribed by clinicians.

Pharmacologic treatments for insomnia have been utilized throughout recorded history [1]. At the start of the twentieth century, barbiturates and related compounds became the most commonly used agents to treat insomnia [1]. The use of barbiturates began to decline in the mid twentieth century when their adverse side effects and the potential for lethal overdose was better understood [1]. In 1963, the first benzodiazepine, namely chlordiazepoxide, was introduced to the United States (US) market [1]. In 1970, flurazepam was the first benzodiazepine approved by the US Food and Drug Administration (FDA) as a hypnotic [1]. Due to a perceived better safety profile, the use of benzodiazepines quickly surpassed that of barbiturates for the treatment of insomnia [1]. In 1992, zolpidem was the first non-benzodiazepine benzodiazepine receptor agonist (nBBRA) hypnotic to become available in the US [1]. To this day, zolpidem remains one of the most widely prescribed medications for the treatment of insomnia [1].

The goal of this review is to describe different classes of medications available in the US for the treatment of insomnia. We will discuss established mechanisms of action, side effect profiles, dosing ranges, and reported efficacy. We will also examine controversies associated with long-term use of hypnotic medication and discuss the treatment of insomnia in special populations. For this article, we reviewed the latest literature from PubMed and Medline as well as practice parameters from the American Academy of Sleep Medicine and other professional organizations.

\section{Neurobiology of Insomnia-Implications for Pharmacotherapy}

While there are multiple psychological models of insomnia, the most commonly accepted one is the 3P model which characterizes etiology based on predisposing, precipitating, and perpetuating factors [12]. When a patient with particular predisposing factors experiences a trigger or precipitant, they develop a maladaptive pattern of coping which leads to the perpetuation of insomnia. This theory forms the basis for CBT-I.

From a neurobiological perspective, sleep is a highly regulated physiologic state with multiple neural substrates for sleep and wakefulness that are extensively distributed throughout the brain (see Fig. 1). The monoaminergic (dopamine, noradrenaline, histamine) and cholinergic systems promote wakefulness, whereas the gamma-aminobutyric acid (GABA)-ergic systems in the pre-optic nucleus and brainstem promote sleep [13]. Several hypnotics exert their effects by acting on $\mathrm{GABA}_{\mathrm{A}}$ receptors. These complex receptors are heterogeneous pentameric membrane proteins that are widely dispersed throughout the central nervous system [1]. They are the target receptors for benzodiazepines, and barbiturates, as well as nBBRAs (Z-drugs). These medications are thought to mediate their anxiolytic, sedative, muscle relaxant, and anticonvulsant effects through GABA-mediated inhibition [2]. Sedative and hypnotic effects appear to be mediated by alpha- 1 and alpha- 2 containing receptor subtypes respectively $[3,6]$. The more recently discovered hypocretin/orexin system regulates wakefulness and suppresses rapid eye movement sleep by providing excitatory input to the monoaminergic and cholinergic systems [14]. These systems interact with the circadian pacemaker located in the suprachiasmatic nucleus to generate normal sleep and wake cycles [15]. Thus, the mechanisms involved in the promotion of sleep and wakefulness are extensive and involve numerous neurotransmitters which offer multiple treatment targets for insomnia.

\section{Hypnotic Use in Clinical Settings}

\section{Long-Term Hypnotic Use}

Insomnia is frequently persistent if left untreated; up to $46 \%$ of patients have insomnia for at least 3 years [16]. Approximately $2.5 \%$ of the US population takes hypnotics for insomnia, of whom $25 \%$ are on nightly medication treatment for greater than or equal to 4 months [17]. Initial guidelines for pharmacotherapy in chronic insomnia recommended the use of medications for up to 4 weeks and were based on studies of short-term hypnotic use with the mean duration of the clinical trials being 1 week [18]. More recent studies have examined the use of hypnotic 


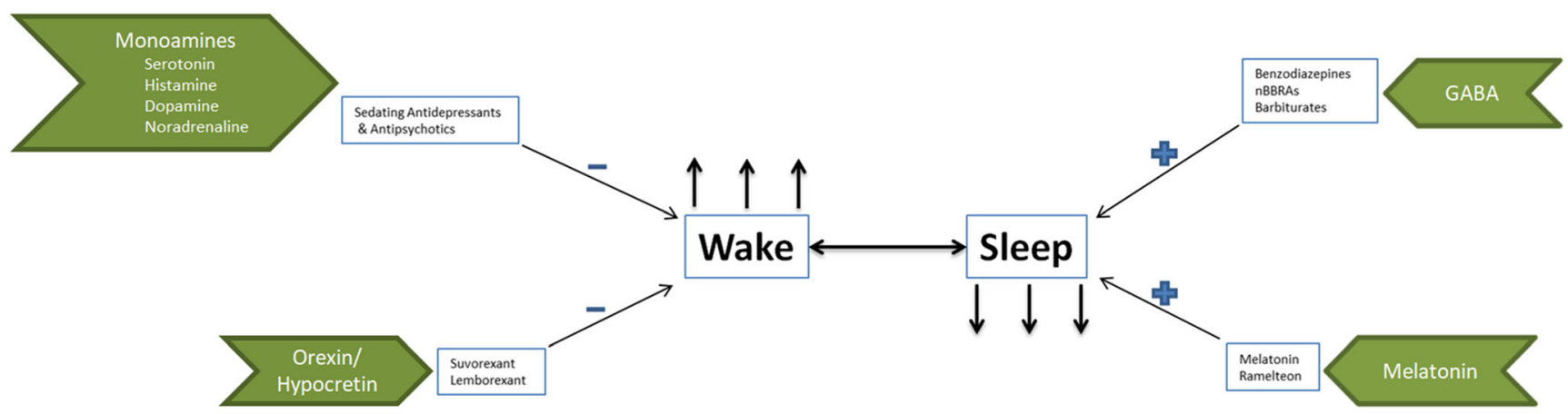

Fig. 1 Neurobiology of sleep and wake and mechanisms of action of hypnotics

medications over a 12-month period and as a result, eszopiclone and zolpidem-extended release (ER) are now FDA-approved for long-term use [19, 20].

Multiple prospective and retrospective studies have linked long-term benzodiazepine and nBBRA hypnotic use with increased risk of falls, psychiatric disorders, cardiovascular disease, dementia, and mortality [21]. Several of these investigations have not addressed or only partially accounted for insomnia severity and a majority are association studies. Additionally, some studies have indicated an increase in benzodiazepine or other hypnotic prescription in the weeks prior to death indicating that these medications are likely prescribed in the context of palliative care [22].

It should be noted that a recent large study reported a lack of association between hypnotic use and dementia in a carefully analyzed large population-based sample [23]. Furthermore, these possible risks have never been formally balanced against the risks of untreated insomnia; there is evidence that insomnia and sleep fragmentation may themselves be risk factors for neurodegenerative diseases including Alzheimer's dementia [24].

\section{Hypnotic Dosing in Women}

In 2013, the FDA required manufacturers to reduce the recommended dose of zolpidem and zolpidem-ER in women and suggested that healthcare providers should consider prescribing lower doses for women [25]. Data from regulatory trials indicated that when the plasma zolpidem level was $>50 \mathrm{mg} / \mathrm{ml}$ $3 \mathrm{~h}$ following the use of sublingual zolpidem, the ability to drive was impaired [25]. Analyses of initial regulatory data involving immediate release zolpidem indicated that up to $15 \%$ of women versus $3 \%$ of men had plasma levels greater than $50 \mathrm{ng} / \mathrm{ml} 8 \mathrm{~h}$ following medication use. The assumption is that due to slower metabolism in women, the plasma levels of the drug the morning after could be high enough to impair driving and other important functions. It should be noted that while recent evidence indicated that up to $35 \%$ of women have a lower apparent clearance of zolpidem, there was no difference between active drug and placebo $8 \mathrm{~h}$ following medication administration on objective tests of functional impairment [26].

\section{Hypnotic Use in Pregnancy}

Pregnancy is a normal physiological event but sleep disruption is extremely common [27]. Poor sleep quality in pregnancy can be a risk factor for depression and is associated with other adverse pregnancy-related outcomes including preterm birth and prolonged labor. In general, pregnant women are excluded from clinical trials and thus data examining the safety and efficacy of hypnotic medications during pregnancy are sparse. A meta-analysis of hypnotic medication-related adverse effects did not reveal any increase in the rates of congenital malformation following the use of these medications in pregnancy [28]. However, benzodiazepines and nBBRAs were associated with preterm birth and low birth weight. Studies examining the use of sedating antihistamines to treat hyperemesis suggest that diphenhydramine and doxylamine are relatively safe in pregnancy [28]. Up to $92 \%$ of pregnant women report that they self-treat sleep disruption with one of these agents [29].

\section{Hypnotic Use in the Elderly}

The prevalence of insomnia is higher in older adults. Changes in body composition, increased comorbidities, polypharmacy, and pharmacokinetics in older adults require more careful prescribing of all medications including hypnotics. Among the nBBRAs, eszopiclone, zolpidem-ER, and zaleplon have been studied in older adults and found to be safe at slightly lower doses [30-32]. Ramelteon and doxepin have also been studied in older adults [33, 34]; the former has been shown to improve sleep onset latency while the latter has been demonstrated to result in a sustained improvement in sleep with minimal adverse effects. Finally, suvorexant has been shown to improve sleep maintenance with minimal associated side effects in this population [35]. 


\section{Hypnotic Use in Patients with Medical Comorbidities}

Most hypnotic agents undergo hepatic and renal clearance. Metabolic clearance of these medications may therefore be delayed in patients who have renal or hepatic disease leading to accumulation and excessive sedation. Patients with hepatic or renal failure should be prescribed the lowest effective does and slowly titrated upwards as tolerated.

Insomnia is commonly comorbid with obstructive sleep apnea (OSA) [36]. While the possibility of hypnotic medications resulting in muscle relaxation thereby worsening OSA is a consideration in clinical settings, studies have shown that eszopiclone and trazodone can result in minimal improvements in sleep apnea severity by increasing the arousal threshold [37, 38]. It is notable that subjects with very severe sleep apnea and oxyhemoglobin desaturation were generally excluded from these trials. Ultimately, it is reasonable to approach comorbid OSA and insomnia independently and in parallel; medications for insomnia, at least in therapeutic doses, need not be withheld in patients with OSA.

\section{Hypnotic Use in Patients with Substance Use Disorders}

Sleep disturbances are commonly comorbid with substance use disorders (SUD) [39]. Up to $80-90 \%$ of individuals in early alcohol recovery experience sleep disruption [40]. Sleep disturbance is also a common symptom in early cannabis withdrawal, with up to $65 \%$ of subjects reporting insomnia. Sleep disturbance occurring in the context of substance use recovery can impact quality of life and increase relapse risk. Data examining the efficacy of hypnotic medications in these circumstances are limited. Trazodone has been shown to improve sleep disruption in alcohol recovery [41]. Studies have demonstrated that zolpidem can result in improvements in sleep following cannabis cessation [42]. However, longer term studies that evaluate the impact of treating sleep disturbance on improved substance use-related outcomes are required. Prescribing hypnotic medications in this population should balance the risk of abuse of these medications against the increased risk of relapse and impaired quality of life secondary to ongoing sleep disturbance.

\section{Hypnotic Medication Classes}

We describe the major hypnotic classes below and summarize their mechanisms of action, dosage, common adverse effects, and contraindications in Table 1.

\section{Benzodiazepine Receptor Agonists}

Eszopiclone, zaleplon, and zolpidem are nBBRAS or Z-drugs that are FDA-approved for the treatment of insomnia. All three medications have been found to be effective and relatively safe in the treatment of insomnia [43].

The exact mechanism of action of eszopiclone is unclear. Dosing of oral eszopiclone ranges from 1 to $3 \mathrm{mg}$. Common side effects of eszopiclone include headache, nausea/emesis, disorder of taste (unpleasant taste), dizziness, somnolence, migraine, and increased respiratory infections. Zaleplon is thought to exert its action through selective binding to alpha subunit of $\mathrm{GABA}_{\mathrm{A}}$ omega-1 receptor. Dosing ranges from 5 to $10 \mathrm{mg}$ orally, with a typical dosage of $10 \mathrm{mg}$, to be administered immediately before bedtime, or for middle of the night awakenings with an additional $4 \mathrm{~h}$ available to sleep. Adverse effects consist of abdominal pain, nausea, headache, dizziness, somnolence, paresthesia, dysmenorrhea, and eye pain. The mechanism of action of zolpidem is selective agonism at the benzodiazepine-1 $\left(\mathrm{BZ}_{1}\right)$ receptor inhibiting action potentials and neuronal excitability. Zolpidem has two main formulations, immediate release dosed at 5-10 $\mathrm{mg}$ and a controlled release preparation dosed at $6.25-12 \mathrm{mg}$; both are administered by mouth immediately prior to bedtime. Intermezzo ${ }^{\mathrm{TM}}$ is a sublingual formulation of zolpidem that was specifically developed for sleep maintenance insomnia, i.e., middle-ofthe-night-wakefulness with difficulty returning to sleep. It is dosed at $1.75-3.5 \mathrm{mg}$ and should be taken only if the patient has an additional $4 \mathrm{~h}$ to sleep. Zolpimist ${ }^{\mathrm{TM}}$ is an oral metered spray containing $5 \mathrm{mg}$ of zolpidem and is used for sleep onset insomnia. Most commonly associated side effects with the use of zolpidem are diarrhea, nausea, dizziness, somnolence, and visual disturbances. All nBBRAs are linked with complex behaviors occurring out of sleep but zolpidem seems to be especially associated with this side effect [44]. Currently, all nBBRAs carry a boxed warning about these potential side effects.

\section{Benzodiazepines}

Benzodiazepines exert their effects as positive allosteric modulators on the $\mathrm{GABA}_{\mathrm{A}}$ binding site that potentiates GABA's inhibitory effect. Through this mechanism, they are thought to produce sedative, anxiolytic, hypnotic, muscle relaxant, and anticonvulsant effects [45]. Currently, there are five benzodiazepines that are FDA-approved for insomnia in the adult population, including estazolam, flurazepam, quazepam, temazepam, and triazolam. Most other benzodiazepines are also associated with sedation and are used off-label as hypnotics quite frequently. Dosing and side effects for these medications are detailed in Table 1. In general, benzodiazepines are associated with ataxia, dizziness, lethargy, sedation, somnolence, apnea, and blurred vision. Although there are reports 


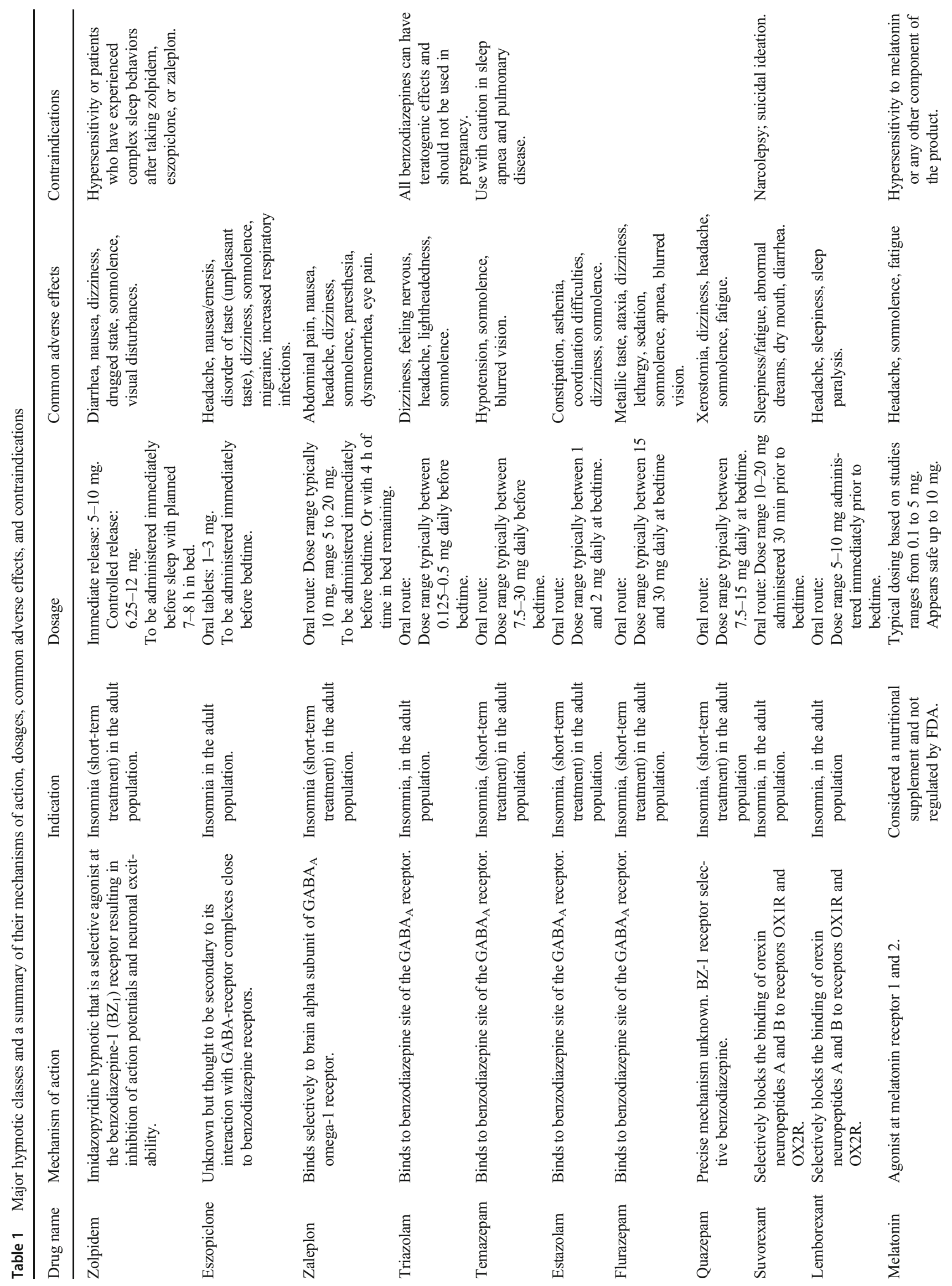


indicating increased risk of anterograde amnesia on triazolam, this can likely occur with other benzodiazepines as well [46, 47].

\section{Dual Orexin Receptor Antagonists}

Orexin neuropeptides A and B and their associated receptors $\mathrm{OX}_{1} \mathrm{R}$ and $\mathrm{OX}_{2} \mathrm{R}$ are critical for sleep-wake regulation [48]. Suvorexant, sold under the trade name Belsomra ${ }^{\mathrm{TM}}$, and lemborexant are dual $\mathrm{OX}_{1} \mathrm{R} / \mathrm{OX}_{2} \mathrm{R}$ antagonists (DORAs) working by selectively blocking the binding of orexin neuropeptides A and B to their respective receptors [49]. This in turn works to suppress wakefulness [49].

Suvorexant was the first in its class to be registered for treatment of insomnia in the US and was approved by the FDA in 2014 [48]. A meta-analysis of 4 trials with a total of 3076 patients showed that suvorexant resulted in improvements in subjective measures of sleep quality [50]. Here, the medication was used at doses of 15-30 mg in the elderly and $30-40 \mathrm{mg}$ in the non-elderly. However, current FDA approval is for a starting dose of $10 \mathrm{mg}$ for most patients, which may be titrated up to $20 \mathrm{mg}$ per day [49]. Major adverse effects include sleepiness/fatigue, abnormal dreams, dry mouth, and diarrhea. There is a concern for increased risk of suicidal ideation with the higher dose regimen [48].

Lemborexant is a more recently approved DORA which has been shown to objectively improve sleep latency, sleep efficiency, and total sleep time [51]. The major side effects include headache, sleepiness, and sleep paralysis.

\section{Sedative Antidepressants}

Doxepin and trazodone are antidepressants that are commonly prescribed as hypnotic agents. Silenor ${ }^{\mathrm{TM}}$ is a newer low-dose formulation (3-6 mg) of the older tricyclic antidepressant doxepin, which is now FDA-approved for insomnia. At this dose, doxepin functions as a pure $\mathrm{H}-1$ receptor antagonist and does not have any of the anticholinergic, antiadrenergic, or serotonergic properties [52]. Doxepin has been shown to be effective in improving sleep maintenance and total sleep duration but with minimal effect on sleep initiation [53]. It has been studied in the elderly population and found to be safe [54]. The main side effects are headache and somnolence [53].

Trazodone is a triazolopyridine which exerts its antidepressant effect through serotonin antagonism and reuptake inhibition [55]. The current American Academy of Sleep Medicine guidelines recommend against its use due to a paucity of evidence on its effectiveness as a hypnotic [1]. Despite this, it appears that trazodone is still commonly used off-label to treat insomnia in doses between 50 and $150 \mathrm{mg}$, especially in patients with psychiatric comorbidity, where there is some evidence to support its efficacy [56-58]. Trazodone is commonly associated with headache and daytime somnolence. Some 
studies have shown a deleterious effect on short-term memory and balance the following morning [59]. Trazodone has been associated with a potentially serious adverse effect of priapism, but this is thought to be rare (risk of 1 in 1000 to 1 in $10,000)[60]$.

\section{Melatonin, Melatonin Receptor Agonists, and Other Supplements}

Melatonin is a hormone that is produced by the pineal gland and is a chronobiotic agent that regulates the sleep-wake cycle [61]. It is a full agonist at melatonin receptors 1 and 2. While melatonin is used to treat circadian rhythm sleep-wake disorders, it is also a weak hypnotic. Meta-analyses of randomized placebo controlled trials have demonstrated improvements in sleep onset latency and total sleep time but these effects are smaller than those of benzodiazepines, nBBRAs, and other hypnotic agents [62]. The dosing regimen for melatonin is unclear, with various studies utilizing doses between 0.1 and $5 \mathrm{mg}$. Melatonin appears to have a benign safety profile with no major adverse effects compared to placebo, at least up to doses of $10 \mathrm{mg}$. Melatonin is considered a nutritional supplement in the US and is not regulated by the FDA. Studies have shown that there is a wide variation in melatonin content compared to that advertised on the label [63].

Ramelteon is a melatonin receptor 1 and 2 agonist. A metaanalysis of 13 trials involving 5812 patients revealed mild improvements in sleep onset latency and sleep quality [64]. There was no change in total sleep time. The main adverse effect noted was somnolence. Ramelteon has been studied in the $>65$-year-old population and found to be safe. However, the overall clinical impact of the medication appears to be small.

While there are multiple nutritional supplements that are marketed for sleep, the most commonly used include valerian root, chamomile, and kava. Valerian root is the most extensively studied in placebo-controlled trials [65]. A metaanalysis of 5 trials did not find any significant difference between valerian root and placebo in sleep-related outcomes. Overall, all of the herbal supplements appear to be relatively safe and well-tolerated with minimal adverse effects.

Diphenhydramine is an antihistaminergic agent that is a common over-the-counter sleep aid. It has been evaluated at a dose of $50 \mathrm{mg}$ in the treatment of chronic insomnia [66]. Minor improvements in subjective and objective sleep measures were noted in this study, not thought to be clinically significant. It is generally not recommended as a hypnotic agent apart from occasional intermittent use in a younger patient, as there are concerns that it can cause daytime grogginess due to its long half-life and result in other significant side effects secondary to its anticholinergic properties.

\section{Barbiturates and Other Older Hypnotics}

Numerous barbiturates have an FDA approval for use as sedative hypnotics. These agents work as agonists and allosteric modulators at the $\mathrm{GABA}_{\mathrm{A}}$ receptor site. However, due to their narrow therapeutic window, potential for abuse/dependence, and significant interactions with alcohol and other CNS depressants, they are rarely used.

Among other older non-barbiturate hypnotic drugs, chloral hydrate is available in the US but is generally not used in clinical practice. Its mechanism of action is not entirely known but it is thought to work through its active metabolite trichloroethanol. Chloral hydrate has a very narrow therapeutic window and is potentially dangerous following an overdose.

\section{Conclusions}

While pharmacotherapy for insomnia is considered secondline treatment, hypnotics are among the most commonly prescribed medications. Current evidence supports the efficacy of these agents in improving sleep quality and quantity. Longterm use of certain hypnotic medications has been shown to be relatively safe. Currently, there are no head-to-head trials indicating a clear hierarchy of efficacy for these agents. The choice of agent, length of treatment, and alternatives should be based on shared decision-making and must weigh the risks and benefits of all possible options in the individual patient.

\section{References}

1. Sateia MJ, Buysse DJ, Krystal AD, Neubauer DN, Heald JL. Clinical Practice Guideline for the Pharmacologic Treatment of Chronic Insomnia in Adults: An American Academy of Sleep Medicine Clinical Practice Guideline. J Clin Sleep Med, 13(2), 307-349 (2017).

2. Bragg S, Benich JJ, Christian N, Visserman J, Freedy J. Updates in insomnia diagnosis and treatment. Int J Psychiatry Med, 54(4-5), 275-289 (2019).

3. Roth T. Insomnia: definition, prevalence, etiology, and consequences. J Clin Sleep Med, 3(5 Suppl), S7-10 (2007).

4. Vgontzas AN, Fernandez-Mendoza J, Liao D, Bixler EO. Insomnia with objective short sleep duration: the most biologically severe phenotype of the disorder. Sleep Med Rev, 17(4), 241-254 (2013).

5. Ford DE, Kamerow DB. Epidemiologic study of sleep disturbances and psychiatric disorders. An opportunity for prevention? JAMA, 262(11), 1479-1484 (1989).

6. Khan MS, Aouad R. The Effects of Insomnia and Sleep Loss on Cardiovascular Disease. Sleep Med Clin, 12(2), 167-177 (2017).

7. Alvaro PK, Roberts RM, Harris JK. A Systematic Review Assessing Bidirectionality between Sleep Disturbances, Anxiety, and Depression. Sleep, 36(7), 1059-1068 (2013).

8. Daley M, Morin CM, LeBlanc M, Gregoire JP, Savard J. The economic burden of insomnia: direct and indirect costs for individuals 
with insomnia syndrome, insomnia symptoms, and good sleepers. Sleep, 32(1), 55-64 (2009).

9. Morgenthaler T, Kramer M, Alessi C et al. Practice parameters for the psychological and behavioral treatment of insomnia: an update. An american academy of sleep medicine report. Sleep, 29(11), 1415-1419 (2006).

10. Haynes J, Talbert M, Fox S, Close E. Cognitive Behavioral Therapy in the Treatment of Insomnia. South Med J, 111(2), 7580 (2018).

11. Mitchell LJ, Bisdounis L, Ballesio A, Omlin X, Kyle SD. The impact of cognitive behavioural therapy for insomnia on objective sleep parameters: A meta-analysis and systematic review. Sleep Med Rev, 47, 90-102 (2019).

12. Spielman AJ, Caruso LS, Glovinsky PB. A behavioral perspective on insomnia treatment. Psychiatr Clin North Am, 10(4), 541-553 (1987).

13. Schwartz MD, Kilduff TS. The Neurobiology of Sleep and Wakefulness. Psychiatr Clin North Am, 38(4), 615-644 (2015).

14. Vazquez-DeRose J, Schwartz MD, Nguyen AT et al. Hypocretin/ orexin antagonism enhances sleep-related adenosine and GABA neurotransmission in rat basal forebrain. Brain Struct Funct, 221(2), 923-940 (2016).

15. Inouye ST, Kawamura H. Persistence of circadian rhythmicity in a mammalian hypothalamic "island" containing the suprachiasmatic nucleus. Proceedings of the National Academy of Sciences, 76(11), 5962-5966 (1979).

16. Morin CM, Bélanger L, LeBlanc $\mathrm{M}$ et al. The natural history of insomnia: a population-based 3-year longitudinal study. Arch Intern Med, 169(5), 447-453 (2009).

17. Balter MB, Uhlenhuth EH. New epidemiologic findings about insomnia and its treatment. J Clin Psychiatry, 53 Suppl, 34-39; discussion 40-32 (1992).

18. National Institutes of Health State of the Science Conference statement on Manifestations and Management of Chronic Insomnia in Adults, June 13-15, 2005. Sleep, 28(9), 1049-1057 (2005).

19. Krystal AD, Erman M, Zammit GK, Soubrane C, Roth T, Group ZS. Long-term efficacy and safety of zolpidem extended-release $12.5 \mathrm{mg}$, administered 3 to 7 nights per week for 24 weeks, in patients with chronic primary insomnia: a 6-month, randomized, double-blind, placebo-controlled, parallel-group, multicenter study. Sleep, 31(1), 79-90 (2008).

20. Krystal AD, Walsh JK, Laska E et al. Sustained efficacy of eszopiclone over 6 months of nightly treatment: results of a randomized, double-blind, placebo-controlled study in adults with chronic insomnia. Sleep, 26(7), 793-799 (2003).

21. Kripke DF. Mortality Risk of Hypnotics: Strengths and Limits of Evidence. Drug Saf, 39(2), 93-107 (2016).

22. Neutel CI, Johansen HL. Association between hypnotics use and increased mortality: causation or confounding? Eur J Clin Pharmacol, 71(5), 637-642 (2015).

23. Osler M, Jørgensen MB. Associations of Benzodiazepines, ZDrugs, and Other Anxiolytics With Subsequent Dementia in Patients With Affective Disorders: A Nationwide Cohort and Nested Case-Control Study. Am J Psychiatry, 177(6), 497-505 (2020).

24. Shamim SA, Warriach ZI, Tariq MA, Rana KF, Malik BH. Insomnia: Risk Factor for Neurodegenerative Diseases. Cureus, 11(10), e6004 (2019).

25. Farkas RH, Unger EF, Temple R. Zolpidem and driving impairment-identifying persons at risk. $N$ Engl J Med, 369(8), 689-691 (2013).

26. Greenblatt DJ, Harmatz JS, Roth T. Zolpidem and Gender: Are Women Really At Risk? J Clin Psychopharmacol, 39(3), 189-199 (2019)

27. Mindell JA, Cook RA, Nikolovski J. Sleep patterns and sleep disturbances across pregnancy. Sleep Med, 16(4), 483-488 (2015).
28. Okun ML, Ebert R, Saini B. A review of sleep-promoting medications used in pregnancy. Am J Obstet Gynecol, 212(4), 428-441 (2015).

29. Black RA, Hill DA. Over-the-counter medications in pregnancy. Am Fam Physician, 67(12), 2517-2524 (2003).

30. Ancoli-Israel S, Krystal AD, McCall WV et al. A 12-week, randomized, double-blind, placebo-controlled study evaluating the effect of eszopiclone $2 \mathrm{mg}$ on sleep/wake function in older adults with primary and comorbid insomnia. Sleep, 33(2), 225-234 (2010).

31. Walsh JK, Soubrane C, Roth T. Efficacy and safety of zolpidem extended release in elderly primary insomnia patients. Am J Geriatr Psychiatry, 16(1), 44-57 (2008).

32. Ancoli-Israel S, Walsh JK, Mangano RM, Fujimori M. Zaleplon, A Novel Nonbenzodiazepine Hypnotic, Effectively Treats Insomnia in Elderly Patients Without Causing Rebound Effects. Prim Care Companion J Clin Psychiatry, 1(4), 114-120 (1999).

33. Roth T, Seiden D, Sainati S, Wang-Weigand S, Zhang J, Zee P. Effects of ramelteon on patient-reported sleep latency in older adults with chronic insomnia. Sleep Med, 7(4), 312-318 (2006).

34. Krystal AD, Durrence HH, Scharf M et al. Efficacy and Safety of Doxepin $1 \mathrm{mg}$ and $3 \mathrm{mg}$ in a 12-week Sleep Laboratory and Outpatient Trial of Elderly Subjects with Chronic Primary Insomnia. Sleep, 33(11), 1553-1561 (2010).

35. Sys J, Van Cleynenbreugel S, Deschodt M, Van der Linden L, Tournoy J. Efficacy and safety of non-benzodiazepine and non-Zdrug hypnotic medication for insomnia in older people: a systematic literature review. Eur J Clin Pharmacol, 76(3), 363-381 (2020).

36. Ong JC, Crawford MR. Insomnia and Obstructive Sleep Apnea. Sleep Med Clin, 8(3), 389-398 (2013).

37. Eckert DJ, Owens RL, Kehlmann GB et al. Eszopiclone increases the respiratory arousal threshold and lowers the apnoea/hypopnoea index in obstructive sleep apnoea patients with a low arousal threshold. Clin Sci (Lond), 120(12), 505-514 (2011).

38. Heinzer RC, White DP, Jordan AS et al. Trazodone increases arousal threshold in obstructive sleep apnoea. Eur Respir J, 31(6), 1308-1312 (2008).

39. Angarita GA, Emadi N, Hodges S, Morgan PT. Sleep abnormalities associated with alcohol, cannabis, cocaine, and opiate use: a comprehensive review. Addict Sci Clin Pract, 11(1), 9 (2016).

40. Kolla BP, Mansukhani MP, Biernacka J, Chakravorty S, Karpyak VM. Sleep disturbances in early alcohol recovery: Prevalence and associations with clinical characteristics and severity of alcohol consumption. Drug Alcohol Depend, 206, 107655 (2020).

41. Kolla BP, Mansukhani MP, Schneekloth T. Pharmacological treatment of insomnia in alcohol recovery: a systematic review. Alcohol Alcohol, 46(5), 578-585 (2011).

42. Vandrey R, Smith MT, McCann UD, Budney AJ, Curran EM. Sleep disturbance and the effects of extended-release zolpidem during cannabis withdrawal. Drug Alcohol Depend, 117(1), 38-44 (2011).

43. Liang L, Huang Y, Xu R, Wei Y, Xiao L, Wang G. Eszopiclone for the treatment of primary insomnia: a systematic review and metaanalysis of double-blind, randomized, placebo-controlled trials. Sleep Medicine, 62, 6-13 (2019).

44. Chen LF, Lin CE, Chou YC, Mao WC, Chen YC, Tzeng NS. A comparison of complex sleep behaviors with two short-acting Zhypnosedative drugs in nonpsychotic patients. Neuropsychiatr Dis Treat, 9, 1159-1162 (2013).

45. Griffin CE, 3rd, Kaye AM, Bueno FR, Kaye AD. Benzodiazepine pharmacology and central nervous system-mediated effects. Ochsner J, 13(2), 214-223 (2013).

46. Morris HH, III, Estes ML. Traveler's Amnesia: Transient Global Amnesia Secondary to Triazolam. JAMA, 258(7), 945-946 (1987).

47. Juhl RP, Daugherty VM, Kroboth PD. Incidence of next-day anterograde amnesia caused by flurazepam hydrochloride and triazolam. Clin Pharm, 3(6), 622-625 (1984). 
48. Clark JW, Brian ML, Drummond SPA, Hoyer D, Jacobson LH. Effects of orexin receptor antagonism on human sleep architecture: A systematic review. Sleep Med Rev, 53, 101332 (2020).

49. Rhyne DN, Anderson SL. Suvorexant in insomnia: efficacy, safety and place in therapy. Ther Adv Drug Saf, 6(5), 189-195 (2015).

50. Kuriyama A, Tabata H. Suvorexant for the treatment of primary insomnia: A systematic review and meta-analysis. Sleep Med Rev, 35, 1-7 (2017).

51. Rosenberg R, Murphy P, Zammit G et al. Comparison of Lemborexant With Placebo and Zolpidem Tartrate Extended Release for the Treatment of Older Adults With Insomnia Disorder: A Phase 3 Randomized Clinical Trial. JAMA Netw Open, 2(12), e1918254 (2019).

52. Goforth HW. Low-dose doxepin for the treatment of insomnia: emerging data. Expert opinion on pharmacotherapy, 10(10), 1649-1655 (2009).

53. Yeung W-F, Chung K-F, Yung K-P, Ng TH-Y. Doxepin for insomnia: A systematic review of randomized placebo-controlled trials. Sleep Medicine Reviews, 19, 75-83 (2015).

54. Lankford A, Rogowski R, Essink B, Ludington E, Heith Durrence $\mathrm{H}$, Roth T. Efficacy and safety of doxepin $6 \mathrm{mg}$ in a four-week outpatient trial of elderly adults with chronic primary insomnia. Sleep Medicine, 13(2), 133-138 (2012).

55. Stahl SM. Mechanism of action of trazodone: a multifunctional drug. CNS spectrums, 14(10), 536-546 (2009).

56. Jaffer KY, Chang T, Vanle B et al. Trazodone for Insomnia: A Systematic Review. Innov Clin Neurosci, 14(7-8), 24-34 (2017).

57. James SP, Mendelson WB. The use of trazodone as a hypnotic: a critical review. J Clin Psychiatry, 65(6), 752-755 (2004).
58. Mendelson WB. A review of the evidence for the efficacy and safety of trazodone in insomnia. J Clin Psychiatry, 66(4), 469476 (2005).

59. Roth AJ, McCall WV, Liguori A. Cognitive, psychomotor and polysomnographic effects of trazodone in primary insomniacs. Journal of sleep research, 20(4), 552-558 (2011).

60. Warner MD, Peabody CA, Whiteford HA, Hollister LE. Trazodone and priapism. Journal of Clinical Psychiatry, 48(6), 244-245 (1987).

61. Arendt J, Skene DJ. Melatonin as a chronobiotic. Sleep Medicine Reviews, 9(1), 25-39 (2005).

62. Ferracioli-Oda E, Qawasmi A, Bloch MH. Meta-analysis: melatonin for the treatment of primary sleep disorders. PLoS One, 8(5), e63773 (2013).

63. Erland LA, Saxena PK. Melatonin Natural Health Products and Supplements: Presence of Serotonin and Significant Variability of Melatonin Content. J Clin Sleep Med, 13(2), 275-281 (2017).

64. Kuriyama A, Honda M, Hayashino Y. Ramelteon for the treatment of insomnia in adults: a systematic review and meta-analysis. Sleep Med, 15(4), 385-392 (2014).

65. Leach MJ, Page AT. Herbal medicine for insomnia: A systematic review and meta-analysis. Sleep Med Rev, 24, 1-12 (2015).

66. Sateia MJ, Buysse DJ, Krystal AD, Neubauer DN, Heald JL. Clinical Practice Guideline for the Pharmacologic Treatment of Chronic Insomnia in Adults: An American Academy of Sleep Medicine Clinical Practice Guideline. J Clin Sleep Med, 13(2), 307-349 (2017).

Publisher's Note Springer Nature remains neutral with regard to jurisdictional claims in published maps and institutional affiliations. 